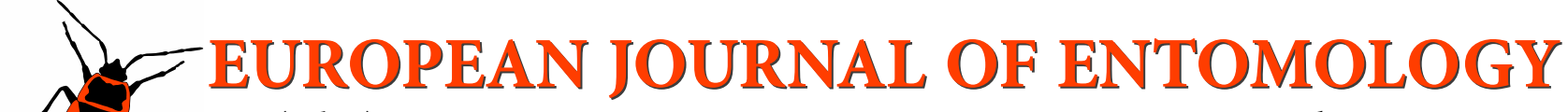 \\ ISSN (online): 1802-8829 \\ http://www.eje.cz \\ Eur. J. Entomol. 117: 210-215, 2020 \\ doi: 10.14411/eje.2020.022 \\ ORIGINAL ARTICLE
}

\section{Laboratory evaluation of effects of powdered sulphur on the oviposition, fruit detection and behaviour of Drosophila suzukii (Diptera: Drosophilidae) on strawberry}

\author{
Sergio PÉreZ-GuerRero ${ }^{1}$, José María MOlina ${ }^{1}$, CRistina MONTIEL $^{1}$, Alberto Redondo-VILLA ${ }^{2}$ \\ and LAURA AVIVAR-LOZANO ${ }^{1}$
}

${ }^{1}$ Laboratorio de Entomología, IFAPA, Centro "Las Torres", Crta. Sevilla-Cazalla de la Sierra, Km 12.2, 41200 Alcalá del Río (Seville), Spain; e-mails: sergio.perez@juntadeandalucia.es, josem.molina@juntadeandalucia.es, cristinamontielp@gmail.com, laura.avivar@juntadeandalucia.es

${ }^{2}$ Departamento de Zoología, Facultad de Ciencias, Universidad de Córdoba, Edificio Charles Darwin, Campus Universitario de Rabanales, Crta. Madrid-Cádiz, Km 396-a, Spain; e-mail: ba1revia@uco.es

Key words. Diptera, Drosophilidae, Drosophila suzukii, sulphur, alternative insecticides, berries, invasive pests, control, fruit coating, organic production

\begin{abstract}
Drosophila suzukii (Matsumura, 1931) is an invasive pest from South-East Asia that can damage a wide range of softskinned fruit crops (especially berries) resulting in serious financial losses. At present, control of $D$. suzukii is still mainly based on adult-oriented chemical pesticides that is likely to result in resistance and environmental pollution. In this context, alternative methods that are compatible with sustainable management are required. Sulphur and its different formulations are widely used for a long time as a fungicide and insecticide on many crops as it is environmentally-friendly and compatible with organic management. In this study, the effect that powdered sulphur-treated strawberries had on oviposition were evaluated under laboratory conditions in order to confirm the findings of a previous report on blueberries. No-choice and choice experiments were carried out to assess whether powdered sulphur affected the ability of the fly to detect fruit, altered its behaviour or did both. The treatment of strawberries resulted in a reduction of over $79 \%$ in the total number of $D$. suzukii that developed. In the no-choice experiment, there was a reduction of over $70 \%$ in the time the flies spent on treated compared to untreated fruit. In the choice experiment, $57 \%$ of the females chose untreated and $43 \%$ treated strawberries, but this difference was not statistically significant. Furthermore, females took over $75 \%$ longer to visit the treated fruit than the control. Sulphur had no noteworthy effect on fly behavior, although oviposition only occurred on untreated fruit indicating that sulphur affects the acceptance and suitability of fruit for females. These results indicate that powdered sulphur could be an appropriate alternative to synthetic-organic insecticides for controlling $D$. suzukii and is compatible with organic crop management and waste reduction strategies.
\end{abstract}

\section{INTRODUCTION}

The Spotted Wing Drosophila, Drosophila suzukii (Matsumura, 1931) (Diptera: Drosophilidae), is a polyphagous invasive pest native to South-East Asia, which affects a wide range of wild plants and important crops in Europe and America. Because it has a serrated ovipositor this species can infest unbruised ripening soft fruit (Asplen et al., 2015). Larvae feed and develop inside the fruit of numerous crops, which becomes unsellable and results in a dramatic reduction in fruit production and financial loss (Goodhue et al., 2011; Walsh et al., 2011; Kinjo et al., 2013). Due to its ability to disperse and polyphagy $D$. suzukii has spread rapidly throughout the world since 2008 when it was first detected in Southern Europe and North America (Lee et al., 2011; Cini et al., 2012). Berries (especially raspberries, blueberries and strawberries) and stone fruit (e.g. cherries, peaches) are the crops most susceptible to infestation. In Andalusia (southern Spain), D. suzukii was first detected in 2012 in the province of Huelva. As over $80 \%$ of the berries in Spain are produced in Huelva, they are an important socio-economic driver in this region. Damage of around 80\% of the strawberry crop, $100 \%$ of the cherry crop and $100 \%$ of organic crops were detected relatively shortly after $D$. suzukii arrived in Spain (Sorribas, 2013).

In conventional farming D. suzukii is mainly chemically controlled in Europe using organophosphorates, pyrethroids and spinosyns. However, using these insecticides has consequences in terms of resistance (Bruck et al., 2011; Smirle et al., 2017; Gress \& Zalom, 2019) and environmental pollution, is detrimental to beneficial arthropods, incompatible with organic management and violates the MRL (maximum residue limit) zero tolerance policy in 
EU berry markets (European Comission, 2019). In recent years, biological methods of control (e.g. parasitoids) and other alternative methods (e.g. plant extracts, push and pull strategies, insect proof-nets, harvest schedule/sanitation) have been increasingly incorporated into integrated pest management programs (Leach et al., 2016, 2018; RossiStacconi et al., 2017; Pérez-Guerrero \& Mateus, 2018), in order to mitigate the consequences of using chemical insecticides and manage this pest in a sustainable manner. Numerous plant-derived extracts and other compounds have been used to deter $D$. suzukii from ovipositing on fruit. Gargani et al. (2013) report the negative effects of Sophora flavescens extract on egg viability on blueberries, Erland et al. (2015) those of Lavandula latifolia and commercial avocado oils and Renkema et al. (2016) the deterrent effect of peppermint oil (among others) and the deterrent and lethal effect of thyme oil on D. suzukii males. Finally, Pérez-Guerrero \& Mateus (2018) recently reported a significant reduction in $D$. suzukii infestation six days after using $S$. flavescens and garlic extracts, and suggest they affected egg viability and/or larval development.

Sulphur and its different formulations have been widely used as fungicides for a long time (e.g. on strawberries for controlling Podosphaera aphanis Wallr.) and as insecticides in a range of crops (e.g. in strawberries, vineyards and tomatoes for controlling Tetranychus urticae Koch and Tuta absoluta Meyrick) because they have little effect on the environment and are compatible with organic management (Williams \& Cooper, 2004; Bloem et al., 2005; Zappalà et al., 2011). However, there has been little research on their effectiveness against $D$. suzukii. Some sulphurbased formulations significantly reduce oviposition on blueberries and strawberries (Pérez-Guerrero \& Molina, 2016; Andreazza et al., 2017b). In the laboratory powdered sulphur on blueberry fruit significantly reduces oviposition and adult emergence by over $76 \%$ and $96 \%$ in no-choice and choice experiments, respectively, and over $40 \%$ adult mortality after a seven day exposure (Pérez-Guerrero \& Molina, 2016). Andreazza et al. (2017b) report a 47.6\% reduction in oviposition and greatly arrested immature development when plants were treated with lime-sulphur. In contrast, powdered sulphur results in low mortality $(<40 \%)$ when used as an insecticide for controlling adults and larvae of D. suzukii and Zaprionus indianus Gupta (Andreazza et al., 2017a). It is known that powdered sulphur deters D. suzukii from ovipositing (Pérez-Guerrero \& Molina, 2016), but the mechanism by which sulphur deters D. suzukii is unknown. Thus, reduction in oviposition may be due either to a deterrent effect of sulphur or it may alter how females behave on fruit.

Our objective was to determine the effects of powdered sulphur on D. suzukii in terms of oviposition, fruit detection and time spent by adults on strawberries in the laboratory using video techniques. First, a choice experiment was carried out to determine whether powdered sulphur inhibits egg-laying on strawberries in order to confirm previous results obtained using blueberries. Second, the time spent on fruit and the behaviour of flies on fruit were analysed using choice and no-choice experiments in order to determine whether powdered sulphur affects their ability to detect fruit, alters their behaviour on fruits or both.

\section{MATERIAL AND METHODS}

\section{Insects, fruit and insecticide}

The D. suzukii adults used in the bioassays came from a colony established at the IFAPA Laboratory of Entomology "Las Torres" (Alcalá del Río, Seville, Spain) from larvae collected from a field of raspberries at Huelva (southern Spain) in February 2014. The colony of $D$. suzukii was continuously reared on berries, mainly blueberries, Vaccinium corymbosum L. (Ericaceae) and raspberries, Rubus idaeus L. (Rosaceae). The D. suzukii adults were kept at a $22 \pm 1{ }^{\circ} \mathrm{C}, 65 \% \mathrm{RH}$, and $16 \mathrm{~L}: 8 \mathrm{D}$ photoperiod in $29 \times 29$ $\times 29 \mathrm{~cm}$ plastic cages (BugDorm ${ }^{\circledR} 1$, Bio-Quip Products Inc., Rancho Rodríguez, CA, USA) with a $25 \mathrm{~mm}$ plastic cup containing a small ball of cotton saturated with $10 \% \mathrm{w} / \mathrm{v}$ honey- $\mathrm{dH}_{2} \mathrm{O}$ as a food source. The female and male $D$. suzukii used in the experiments were between 5 and 12 days old and had no contact with fruit from the time they emerged until they were used in the experiments. The substance tested was powdered sulphur $(98.5 \%$ DP formulation, Bago d'Ouro ${ }^{\circledR}$, Sapec Agro, Valencia, Spain). Ripe commercial strawberries Var. "San Andrea" (FresDoñarosa, Superexport Cia. Agraria S.L., Huelva, Spain) were used in the experiments.

\section{Effects on oviposition}

A choice experiment was used to determine the deterrent effect of powdered sulphur on D. suzukii oviposition. Prior to the experiment strawberries were rinsed with distilled water and then allowed to dry for $2 \mathrm{~h}$. Strawberries were placed in a $0.036 \mathrm{~mm}$ nylon mesh sieve (Filtra Vibración S.L.; Barcelona, Spain) and powdered sulphur was applied directly to them (about $1 \mathrm{mg}$ per fruit); then they were gently rolled by tilting the mesh to prevent damage and to ensure that the entire surface of each fruit was covered. The fruit was shaken carefully so that all surplus powdered sulphur fell through the mesh (Pérez-Guerrero \& Molina, 2016). The dose per fruit was estimated by weighing 10 groups of 10 fruit beforehand, and this was $0.19 \pm 0.002 \mathrm{~g}$ of sulphur per fruit after treatment. All treated fruit were kept under laboratory conditions $\left(22 \pm 1{ }^{\circ} \mathrm{C}, 60 \pm 5 \% \mathrm{RH}\right.$, and a $16 \mathrm{~L}: 8 \mathrm{D}$ photoperiod $)$ for $1 \mathrm{~h}$ before exposure to $D$. suzukii. The fruit were exposed to $D$. suzukii in $29 \times 29 \times 29 \mathrm{~cm}$ plastic cages (BugDorm ${ }^{\circledR} 1$, Bio-Quip Products Inc., Rancho Rodríguez, CA, USA). Two strawberries (treated and untreated) were placed on the floor of the cage in alternating order in each of the replicates (to eliminate any bias). The strawberries were spaced $4 \mathrm{~cm}$ apart and $5 \mathrm{~cm}$ from the food source (a $25 \mathrm{~mm}$ plastic cup containing a small ball of cotton saturated with $10 \% \mathrm{w} / \mathrm{v}$ honey- $\mathrm{dH}_{2} \mathrm{O}$ ). Two females and two males per cage were exposed to two strawberries for $24 \mathrm{~h}$. No adults died during the experiments. After this time, the strawberries were removed from the cages and each fruit was kept separately and incubated under laboratory conditions. D. suzukii eggs are difficult to see on structured fruit such as blackberries, raspberries and strawberries, which renders egg counts unreliable (Lee et al., 2011), so the strawberries were dissected seven days after exposure and the number of larvae and pupae counted. To quantify the larvae and pupae per fruit, each strawberry was placed in a plastic bag with salt water $(5 \%-6 \% \mathrm{v} / \mathrm{v})$. The strawberries were lightly crushed and left for at least $1 \mathrm{~h}$ before the contents of the bag were transferred to a container with a dark background and the $D$. suzukii larvae and pupae were counted under a dissecting microscope (Van Timmeren \& Isaacs, 2013; Pérez-Guerrero \& Mateus, 2018). 


\section{Effects on fruit detection and behaviour}

A no-choice experiment was used to analyse the potential effects of powdered sulphur on the ability of the $D$. suzukii to detect fruit. The strawberries were treated as above and a treated or untreated strawberry was placed in $29 \times 29 \times 29 \mathrm{~cm}$ plastic cages (BugDorm ${ }^{\circledR}$ 1, Bio-Quip Products Inc., Rancho Rodríguez, CA, USA) with one female and one male D. suzukii, and kept at a 22 $\pm 1^{\circ} \mathrm{C}, 60 \pm 5 \% \mathrm{RH}$, and a $16 \mathrm{~L}: 8 \mathrm{D}$ photoperiod. The flies were observed for 30 mins and the time they took to first visit the fruit, the number of visits and time they spent on the fruit were recorded for the males and females using a digital stopwatch. After 30 mins, the strawberries were removed from the cages and each of them was incubated separately under laboratory conditions. Each treatment in this experiment was replicated 25 times over 1-9 days by three observers.

Finally, a choice experiment was used to evaluate the effects of powdered sulphur on the behaviour of females. One treated and one untreated strawberry were placed on $4 \mathrm{~mm}$-diameter plastic rings and placed in the experimental arena, which was a $12 \times$ $12 \times 12 \mathrm{~cm}$ glass cube. The strawberries were $4 \mathrm{~cm}$ from each other and one female was placed in the centre of the arena and filmed for 15 min using two digital video cameras (Canon HFS 21), which together were focused on the entire surface of the fruit. The images were analysed using the Adobe Premiere Pro CS5 video program. Four categories were used to define the behaviour of the fly : (1) walking, the fly takes steps with interruptions $<1$ s; (2) cleaning, the fly does not move and rubs its legs together or its head, wings or ovipositor; (3) oviposition, the fly probes the skin of the strawberry with its ovipositor and (4) stationary, the fly does not move or behave in any of the ways described above (Lemoyne et al., 2008). In addition, the time that elapsed until the first visit to the fruit, the time spent on the fruit and the number of visits were recorded. There was a total of 25 replicates of this experiment.

\section{Statistical procedures}

Since residuals were not normally distributed in the choice and no choice experiments, generalized linear mixed models (GLMMs) were used to determine the effects of the treatment on the number of $D$. suzukii that developed using the "glmer" function in the R v.3.1.3 software package. The GLMMs included treatment as a fixed factor and total $D$. suzukii larvae and pupae as dependent variables fitted to a Poisson distribution with a log-link function. Since treated and untreated fruit were in the same cage, replicate (ID test) was included as a random factor and treatment as a random slope by adding it to the random effects structure. Therefore, the model estimates a random intercept, random slope, the correlation between the two and the fixed effect of treatment (Harrison et al., 2018). The data from the second ("no choice") experiment were analysed in the same way: GLMMs with interaction terms, which included treatment (untreated and treated) and sex as fixed factors and time spent on and off the fruit or the time that elapsed until the first visit to the fruit and from then until the end of the experiment (using the cbind function) as dependent variables fitted to a binomial distribution with a logit link function or number of visits as dependent variables fitted to a Poisson distribution with a log-link function. In order to incorporate male-female variability, replicate (ID test) was considered to be a random factor and sex was specified as a random slope by adding it to the random effects structure. Data from the third experiment ("choice") were also analysed using GLMMs: first, treatment was included as a fixed factor and time spent on and off fruit or time that elapsed before the first visit to the fruit and then to the end of the experiment (using the cbind function) were dependent variables fitted to a binomial distribution with a logit link function.

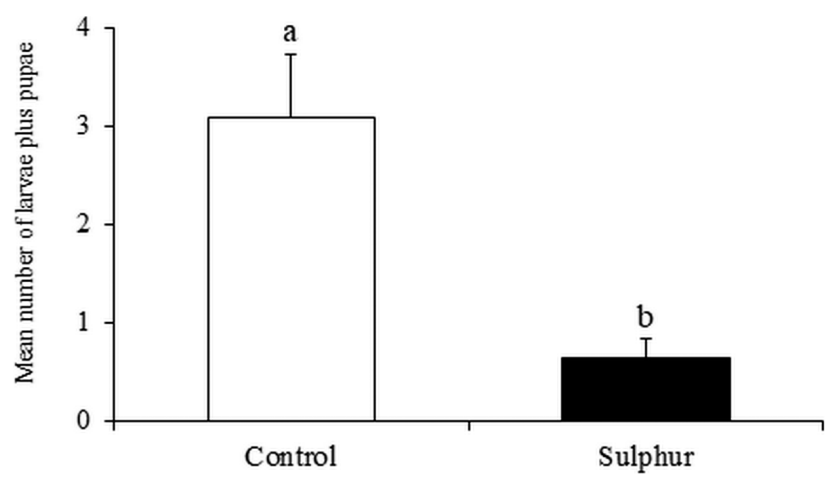

Fig. 1. Mean number of larvae plus pupae of Drosophila suzukii that developed per untreated and treated fruit (control and sulphur) during seven days after $24 \mathrm{~h}$ of exposure to two male-female couples of $D$. suzukii in a choice experiment. Error bars indicate standard error of the mean and different letters denote a significant difference based on generalized linear mixed models (GLMMs) between control and treatment in which alpha $=0.05$.

Secondly, the treatment was included as a fixed factor and time spent on and off the fruit for each behavioural category (using the cbind function) were dependent variables fitted to a binomial distribution with a logit link function. As in the first experiment, replicate (ID test) was considered as a random factor and treatment was specified as a random slope by adding it to the random effects structure. We checked the models for overdispersion and residual distribution using the DHARMa package. When overdispersion was detected, the models were fitted to a quasibinomial or quasipoisson distribution using the "glmmPQL" function. GLMMs procedures used the Wald statistic (" $z$ ") value and $\operatorname{Pr}([|z|])$ or " $t$ " and $\operatorname{Pr}([|t|])$ (when dispersion is estimated) in order to analyse the effects each factor has on the response variable, testing the hypothesis that the corresponding parameter (regression coefficient) is zero (Crawley, 2005). Finally, the number of females that first visited the treated and untreated fruit were compared using Pearson's $\chi^{2}$ test.

\section{RESULTS}

\section{Effects on oviposition}

There was a significant difference in the number of $D$. suzukii larvae and pupae recorded in the treatments $(\mathrm{z}=$ $2.49 ; \mathrm{P}=0.0125)$. The number of larvae and pupae in treat-

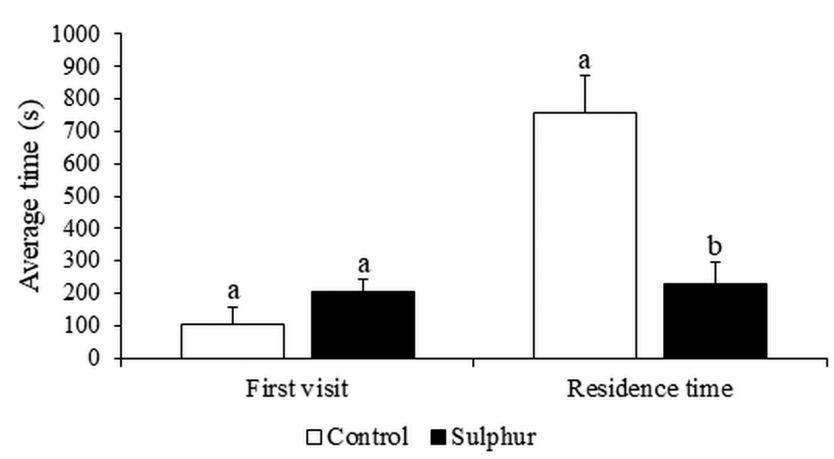

Fig. 2. Average time to the first visit and average time spent on treated and untreated fruit by adults (males and females) of Drosophila suzukii in a no-choice experiment that lasted for $30 \mathrm{~min}$. Error bars indicate standard error of the mean and different letters denote a significant difference based on generalized linear mixed models (GLMMs) between control and treatment in which alpha $=$ 0.05 . 


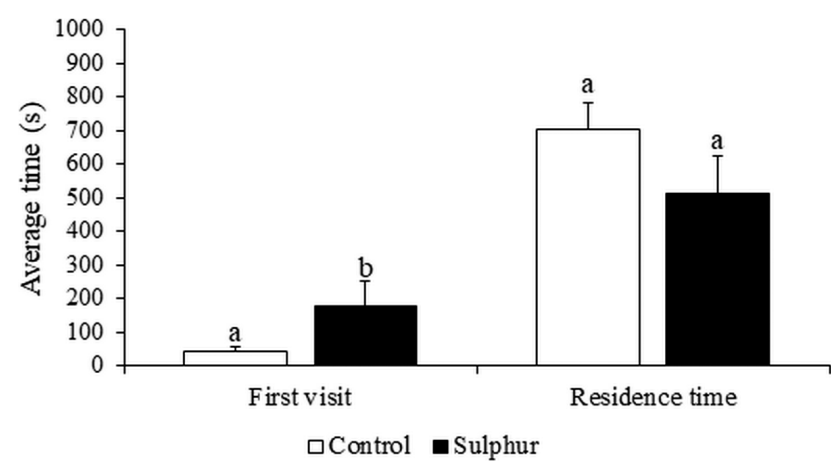

Fig. 3. Average time to the first visit and average time spent on untreated and treated fruit by females of Drosophila suzukii in a choice experiment that lasted for $15 \mathrm{~min}$. Error bars indicate standard error of the mean and different letters denote a significant difference based on generalized linear mixed models (GLMMs) between control and treatment in which alpha $=0.05$.

ed fruit $(0.64 \pm 0.20)$ was reduced by $79 \%$ compared to untreated fruit (3.09 \pm 0.65 ; Fig. 1).

\section{Effects on fruit detection and behaviour}

There were no significant differences $(\mathrm{z}=0.95 ; \mathrm{P}>0.05)$ in the mean number of visits to treated $(0.72 \pm 0.14)$ and untreated fruit $(0.52 \pm 0.08)$ in the no-choice experiment. The GLMMs also revealed no significant differences $(\mathrm{z}=$ $-0.62 ; \mathrm{P}>0.05)$ between males $(0.52 \pm 0.10)$ and females $(0.72 \pm 0.12)$ and in the interaction between these factors $(\mathrm{z}$ $=-0.04 ; \mathrm{P}>0.05)$. Likewise, there were no significant differences in the time that elapsed before the first visit to the fruit among the treatments $(t=0.85 ; \mathrm{P}>0.05$; Fig. 2$)$, by males and females $(t=-1.66 ; P>0.05)$ and the interaction between these factors $(t=-0.67 ; P>0.05)$. In contrast, the GLMMs revealed significant differences in the time spent on the fruit among treatments $(t=5.61 ; \mathrm{P}<0.001)$. There was a reduction of over $70 \%$ in the time the flies spent on treated than on untreated strawberries $(229.02 \pm 65.12 \mathrm{~s}$ and $755.72 \pm 117.37$ s, respectively; Fig. 2). In addition, there were no significant differences in the time spent on the fruit by males and females $(\mathrm{t}=1.50 ; \mathrm{P}>0.05)$ and their interactions $(\mathrm{t}=-0.70 ; \mathrm{P}>0.05)$.

As with the no-choice experiment, no differences were recorded in the choice experiment regarding which fruit ( $42.86 \%$ for treated and $57.14 \%$ for untreated or control) was visited first by females $\left(\chi_{(1)}^{2}=0.86 ; \mathrm{P}>0.05\right)$. The females took over $75 \%$ longer $(175.75 \pm 74.68 \mathrm{~s})$ to visit treated fruit $(\mathrm{z}=-279 ; \mathrm{P}=0.005$; Fig. 3) than the control $(44.00 \pm 14.54 \mathrm{~s})$. In contrast, there were no significant differences in the time spent on the untreated and treated strawberries $(z=1.02 ; P=0.049 ;$ Fig. 3$)$. Furthermore, the GLMMs revealed no significant differences between the treatment and control in terms of the time spent on the fruit in each behavioural category (Table 1). There were no significant differences between the control and treated fruit in the time the flies spent walking, stationary and cleaning $(\mathrm{z}=-0.69 ; \mathrm{P}>0.05 ; \mathrm{z}=1.19 ; \mathrm{P}>0.05$ and $\mathrm{z}=-1.01$; $\mathrm{P}>0.05$, respectively). Finally, although oviposition only occurred on control fruit (Table 1), GLMMs revealed no
Table 1. Average $( \pm S E)$ and percentage $(\%)$ of the time females of Drosophila suzukii spent in each of the defined behavioural categories (see Material and Methods section) when simultaneously exposed to treated and untreated (control) fruit in a choice experiment for $15 \mathrm{~min}$. Different letters denote a significant difference based on generalized linear mixed models (GLMMs) between control and treatment (alpha $=0.05$ ).

\begin{tabular}{lcc}
\hline \multirow{2}{*}{ Behaviour } & \multicolumn{2}{c}{ Actual time spent (s) (\% of time spent) } \\
\cline { 2 - 3 } Stationary & $555.83 \pm 73.76$ & Sulphur \\
& $(62.84 \pm 7.34 \mathrm{a})$ & $(57.87 \pm 13.20 \mathrm{a})$ \\
Walking & $147.58 \pm 39.97$ & $51.63 \pm 27.60$ \\
& $(22.44 \pm 5.50 \mathrm{a})$ & $(22.37 \pm 12.09 \mathrm{a})$ \\
Cleaning & $83.42 \pm 18.32$ & $142.50 \pm 105.81$ \\
& $(13.41 \pm 3.71 \mathrm{a})$ & $(19.82 \pm 7.29 \mathrm{a})$ \\
Ovipositing & $7.83 \pm 7.53$ & 0.00 \\
& $(0.79 \pm 1.60 \mathrm{a})$ & $(0.00 \mathrm{a})$ \\
\hline
\end{tabular}

significant differences with respect to the treated fruit $(\mathrm{t}=$ $-0.009 ; \mathrm{P}>0.05)$.

\section{DISCUSSION}

The results for strawberries support the previous report by Pérez-Guerrero \& Molina (2016) for blueberries in which the infestation of fruit treated with powdered sulphur was significantly less. Treatment of strawberries resulted in a reduction of over $79 \%$ in the number of $D$. suzukii that developed. There are few studies on how sulphur affects D. suzukii (Pérez-Guerrero \& Molina, 2016; Andreazza et al., 2017a,b). Das et al. (2010) report that the sulphur-based fungicide Thiovit affects several life history traits of Drosophila melanogaster resulting in a slightly lower infestation. However, it is comparable to the effect of the treatment of blueberries with same sulphur formulation on eggs laid and adults affected (98 and 96\%, respectively) under similar conditions (Pérez-Guerrero \& Molina, 2016). In this study, the reduction estimates for strawberries were based on the number of larvae and pupae per fruit after seven days. Powdered sulphur is more effective than other formulations (Andreazza et al., 2017b). In fact, Andreazza et al. (2017b) report a significant $47.6 \%$ reduction in oviposition and marked slowdown in immature development when plants were treated with lime-sulphur. However, when powdered sulphur was used as an insecticidal bait it did not control the adults and larvae of $D$. suzukii and $Z$. indianus as the mortality was less than $40 \%$ (Andreazza et al., 2017a).

Powdered sulphur is highly effective in controlling $D$. suzukii attacking strawberries and could be used instead of pesticides (e.g. organophosphorates, pyrethroids and spinosyns) in conventional production. In addition, powered sulphur could help control this pest in organic production (powdered sulphur is currently authorized as a fungicide for organic production of susceptible crops such as strawberries, and farmers use it at least once or twice a year at a rate of $20-30 \mathrm{~kg}$ per hectare). Furthermore, field observations indicate that the lower effect of $D$. suzukii on strawberry crops in southern Spain (grown intensively in large plastic tunnels from October-November and May-June) may be due to the widespread use of powdered sulphur 
during the fruiting season for deterring other pests and preventing plant diseases (Molina, 2015). However, it must be stressed that further research is required in order to evaluate how the residue of sulphur dust affects the pre-harvest and sale of treated fruit in order to define commercially viable strategies for managing the different crops attacked by D. suzukii (Pérez-Guerrero \& Molina, 2016).

Results obtained in the no-choice experiment showed that D. suzukii spent significantly less time on treated fruit than on the control, which may be due to the toxic and deterrent effects of sulphur (Williams \& Cooper, 2004). It is reported that powdered sulphur remains lethal for adults for seven days (not tested here), especially males confined in small experimental chambers (Pérez-Guerrero \& Molina, 2016). Thus, it seems that powdered sulphur acts as a repellent and reduces the ability of $D$. suzukii to detect and oviposit on treated fruit. Furthermore, females in the choice experiment took significantly longer to detect fruit treated with powdered sulphur than control fruit. Like most frugivorous insects, D. suzukii seems to depend on visual and olfactory cues for detecting suitable hosts for oviposition (Keesey et al., 2015; Little et al., 2018). In addition, some authors suggest that $D$. suzukii are mainly attracted by the colour red (Renkema et al., 2014; Kirkpatrick et al., 2016; Rice et al., 2016). Sulphur-covered fruit could affect these visual and olfactory cues, thereby hindering the flies ability to detect fruit. Lemoyne et al. (2008) report changes in the intensity (from darker to lighter) and hue of the colour (from indigo-blue to turquoise-blue) of blueberries treated with kaolin, which affects the ability of blueberry maggot (Rhagoletis mendax Curran) to detect and oviposit on blueberries. Sulphur did not significantly affect how $D$. suzukii behaved on fruit. Lemoyne et al.'s (2008) study of how coming into contact with kaolin affects $R$. medax searching for blueberries report it did not affect cleaning behaviour and oviposition. According to these authors, oviposition in the choice experiment was little affected, which would explain why no significant differences were recorded. However, it should be noted that they only oviposit on untreated fruit, which indicates that sulphur influences whether females consider fruit to be acceptable or suitable. This may account for the reduction in infestation reported here for strawberries and previously for blueberries (Pérez-Guerrero \& Molina, 2016). Nevertheless, more extensive studies are needed to confirm that powdered sulphur directly affects $D$. suzukii oviposition. Finally, it must be stressed that, since the exposure of flies to sulphur may vary greatly under field conditions, the current experiment may be considered a best-case situation as sulphur completely covered the fruit in our laboratory experiment.

In conclusion, laboratory experiments revealed that powdered sulphur can significantly reduce the infestation of strawberries, thereby confirming previous results for blueberries. This sulphur formulation also affected the ability of the D. suzukii to detect fruit and the time they remained in contact with it; hence the lower probability of oviposition on treated fruit. Powdered sulphur seems to be a suitable alternative to synthetic-organic and neurotoxic insecticides. In addition, its use is compatible with organic crop management and waste reduction strategies. Field research is needed to confirm the findings of this study and determine how best to use sulphur as an insecticide for protecting strawberries (where sulphur is currently used as a fungicide) and other berries.

ACKNOWLEDGEMENTS. This work was funded by IFAPA, project PP. AVA.AVA.201601.10 (objective 2). S. Pérez-Guerrero was supported by the Operational Program from the European Social Fund 2007-2013 from Andalusia, Core theme (increase and improvement in human capital). L. Avivar-Lozano was partially supported by the project BACO (CGL2015-68220-R) funded by The Spanish Ministry of Economy, Industry and Competitivity. The authors would like to thank E. Vargas-Osuna and H.K. Aldebis for their help during this study.

\section{REFERENCES}

Andreazza F., Bernardi D., Baronio C.A., Pasinato J., Nava D.E. \& Botтon M. 2017a: Toxicities and effects of insecticidal toxic baits to control Drosophila suzukii and Zaprionus indianus (Diptera: Drosophilidae). — Pest Manag. Sci. 71: 146-152.

Andreazza F., Vacacela A.K., Haddi F., Colares A., Pallini A. \& Oliveira E.E. 2017b: Toxicity to and egg-laying avoidance of Drosophila suzukii (Diptera: Drosophilidae) caused by an old alternative inorganic insecticide preparation. - Pest Manag. Sci. 74: 861-867.

Asplen M.K., Anfora G., Biondi A., Choi D.S., Chu D., DaAne K.M., Gibert P., Gutierrez A.P., Hoelmer K.A, Hutchison W.D. ET AL. 2015: Invasion biology of spotted wing Drosophila (Drosophila suzukii): a global perspective and future priorities. - J. Pest Sci. 88: 469-494.

Bloem E., Haneklaus S. \& Schnug E. 2005: Significance of sulfur compounds in the protection of plants against pests and diseases. - J. Plant Nutr. 28: 763-784.

Bruck D.J., Bolda M., Tanigoshi L., Klick J., Kleiber J., DeFrancesco J., Gerdeman B. \& Spitler H. 2011: Laboratory and field comparisons of insecticides to reduce infestation of Drosophila suzukii in berry crops. - Pest Manag. Sci. 67: 1375-1385.

Cini A., Ioriatti C. \& Anfora G. 2012: A review of the invasion of Drosophila suzuki in Europe and a draft research agenda for integrated pest management. — Bull. Insectol. 65: 49-60.

CraWley M.J. 2005: Statistics: An Introduction Using R. Wiley, Chichester, 327 pp.

Das S., Podder S. \& Roy S. 2010: Effect of fungicide, Thiovit ${ }^{\circledR}$ jet on several life history trait of Drosophila melanogaster (Diptera: Drosophilidae). - J. Appl. Biol. Sci. 4: 31-36.

ERLand L.A., Rheault M.R \& Mahmoud S.S. 2015: Insecticidal and oviposition deterrent effects of essential oils and their constituents against the invasive pest Drosophila suzukii (Matsumura) (Diptera: Drosophilidae). - Crop Prot. 78: 20-26.

European Comission 2019: EU - Pesticide Database (MRLs). URL: https://ec.europa.eu/food/plant/pesticides/eu-pesticidesdatabase (last accesed 7 Nov. 2019).

Gargani E., Tarchi F., Frosinini R., Mazza G. \& Simoni S. 2013: Notes on Drosophila suzukii Matsumura (Diptera Drosophilidae): field survey in Tuscany and laboratory evaluation of organic products. - Redia 96: 85-90.

Goodhue R.E., Bolda M., Farnsworth D., Williams J.C \& ZALOM F.G. 2011: Spotted wing Drosophila infestation of California strawberries and raspberries: Economic analysis of po- 
tential revenue losses and control costs. - Pest Manag. Sci. 67: 1396-1402.

Gress B.E. \& Zalom F.G. 2019: Identification and risk assessment of spinosad resistance in a California population of Drosophila suzukii. - Pest Manag. Sci. 75: 1270-1276.

Harrison X.A., Donaldson L., Correa-Cano M.E., Evans J., Fisher D.N., Goodwin C.E.D., Robinson B.S., Hodgson D.J. \& INGER R. 2018: A brief introduction to mixed effects modelling and multi-model inference in ecology. - Peer J. 6: e4794, $32 \mathrm{pp}$.

Keesey I.W., Knaden M. \& Hansson B.S. 2015: Olfactory specialization in Drosophila suzukii supports an ecological shift in host preference from rotten to fresh fruit. -J. Chem. Ecol. 41: $121-128$.

Kinjo H., Kunimi Y., Ban T. \& NaKai M. 2013: Oviposition efficacy of Drosophila suzukii (Diptera: Drosophilidae) on different cultivars of blueberry. - J. Econ. Entomol. 106: 1767-1771.

KirkPatrick D.M., McGhee P.S., Hermann S.L., Gut L.J. \& MillER J.R. 2016: Alightment of spotted wing Drosophila (Diptera: Drosophilidae) on odorless disks varying in color. - Environ. Entomol. 45: 185-191.

Leach H., Van Timmeren S. \& Isaacs R. 2016: Exclusion netting delays and reduces Drosophila suzukii (Diptera: Drosophilidae) infestation in raspberries. - J. Econ. Entomol. 109: 2151-2158.

Leach H., Moses J., Hanson E., Fanning P. \& Isaacs R. 2018 Rapid harvest schedules and fruit removal as non-chemical approaches for managing spotted wing Drosophila. - J. Pest Sci. 91: $219-226$

Lee J.C., Bruck D.J., Curry H., Edwards D., Haviland D.R., Van Steenwyk R.A. \& Yorgey B.M. 2011: The susceptibility of small fruits and cherries to the spotted-wing Drosophila, Drosophila suzukii. - Pest Manag. Sci. 67: 1358-1367.

Lemoyne P., Vincent C., Gaul S. \& Mackenzie K. 2008: Kaolin affects blueberry maggot behavior on fruit. - J. Econ. Entomol. 101: 118-125.

Little C.M., Chapman T.W. \& Hillier N.K. 2018: Effect of color and contrast of highbush blueberries to host-finding behavior by Drosophila suzukii (Diptera: Drosophilidae). — Environ. Entomol. 47: 1242-1251.

Molina J.M. 2015: Drosophila suzukii (Matsumra, 1391) (Diptera, Drosophilidae) en la fresa de Huelva. - Phytoma España 269: 56-58.

Pérez-Guerrero S. \& Mateus C. 2018: Field evaluation of commercial plant extracts against Drosophila suzukii (Diptera: Drosophlidae) in raspberry. — Int. J. Pest Manag. 65: 53-58.
Pérez-Guerrero S. \& Molina J.M. 2016: Laboratory approach to the use of sulphur and kaolin as preventive control against Drosophila suzukii. - Span. J. Agric. Res. 14(2): e10SC01, $6 \mathrm{pp}$.

Renkema J.M., Buitenhuis R. \& Hallett R.H. 2014: Optimizing trap design and trapping protocols for Drosophila suzukii (Diptera: Drosophilidae). - J. Econ. Entomol. 107: 2107-2118.

Renkema J.M., Wright D., Buitenhuis R. \& Hallett R.H. 2016: Plant essential oils and potassium metabisulfite as repellents for Drosophila suzukii (Diptera: Drosophilidae). — Sci. Rep. 6: $21432,10 \mathrm{pp}$.

Rice K.B., Short B.D., Jones S.K. \& Leskey T.C. 2016: Behavioral responses of Drosophila suzukii (Diptera: Drosophilidae) to visual stimuli under laboratory, semifield, and field conditions. - Environ. Entomol. 45: 1480-1488.

Rossi-Stacconi M.V., Panel A., Baser N., Ioriatti C., Pantezzi T. \& ANFora G. 2017: Comparative life history traits of indigenous Italian parasitoids of Drosophila suzukii and their effectiveness at different temperatures. - Biol. Contr. 112: 20-27.

Smirle M.J., Zurowski C.L., Ayyanath M.M., Scott I.M. \& MacKenZIE K.E. 2017: Laboratory studies of insecticide efficacy and resistance in Drosophila suzukii (Matsumura) (Diptera: Drosophilidae) populations from British Columbia, Canada. Pest Manag. Sci. 73: 130-137.

Sorribas R. 2013: Mecanismos de control para Drosophila suzukii, dentro de la GIP. In: III Jornadas Internacionales sobre feromonas, atrayentes, trampas y control biológico: herramientas para la gestión integrada. - Phytoma (Spain) No. 254: 47.

VAN Timmeren S. \& IsaACS R. 2013: Control of spotted wing Drosophila, Drosophila suzukii, by specific insecticides and by conventional and organic crop protection pro-grams. - Crop Prot. 54: 126-133.

Walsh D.B., Bolda M.P., Goodhue R.E., Dreeves A.J., Lee J.C., Bruck D.J., Walton V.M., O’Neal S.D. \& Zalom F.G. 2011: Drosophila suzukii (Diptera: Drosophilidae): invasive pest of ripening soft fruit expanding its geographic range and damage potential. - J. Integr. Pest Manag. 1: 1-7.

WiLLIAMs J.S. \& COOPER R.M. 2004: The oldest fungicide and newest phytoalexin - A reappraisal of the fungitoxicity of elemental sulphur. - Plant Pathol. 53: 263-279.

Zappalà L., Siscaro G., Biondi A., Mollá O., González-Cabrera J. \& URBAneJA A. 2011: Efficacy of sulphur on Tuta absoluta and its side effects on the predator Nesidiocoris tenuis. - J. Appl. Entomol. 136: 401-409.

Received September 10, 2019; revised and accepted April 2, 2020 Published online April 28, 2020 